\title{
Experiencia de implantación de un método docente constructivista en la enseñanza de la medicina legal. La libertad de prescripción como ejemplo
}

\author{
F. Francés-Bozal, A. Castelló-Ponce, F. Verdú-Pascual
}

Introducción. El método constructivista va implantándose lentamente en la formación médica. En este tipo de aprendizaje, es el alumno el que va a construir el conocimiento sobre las bases cognitivas que ya posee. El profesor que sigue este método no se limita a suministrar un conocimiento ya elaborado, sino que ha de dirigir al alumno en su proceso autónomo y activo de aprendizaje. La principal limitación es el tiempo necesario para llevarlo a cabo con éxito porque obviamente el alumno necesita una información previa y un tiempo de elaboración y procesamiento de esos datos para conseguir construir un aprendizaje duradero. En el texto que sigue se describe la experiencia de la aplicación del método constructivista a la enseñanza de la medicina legal. Materiales y métodos. Se ha utilizado uno de los seminarios programados en la asignatura (prescripción médica) para ensayar las posibilidades de este método de aprendizaje en medicina y determinar su validez. Resultados y conclusiones. Los resultados muestran que el método, en cualquiera de sus modalidades, es especialmente idóneo para el estudiante profesional médico porque obliga a una integración eficiente de la información recibida y la elaboración de unos supuestos que serán sometidos a una revisión crítica, lo que será de aplicación en la práctica diaria de su profesión.

Palabras clave. Educación. Estudiantes. Medicina. Método constructivista.

\section{Implementation of a constructivist teaching method for the learning of legal medicine. Freedom of prescription as example}

Introduction. The constructivist learning method is being slowly implanted in medical training. In this type of learning, the student builds his knowledge from the cognitive founda- tions that already has. The professor who follows this method does not limit itself to provide an elaborated knowledge, but must lead the students in their autonomous and active learning process. The main limitation of this method is the time needed to carry it out successfully because the student needs previous information and a time to prepare and to process the provided data in order to build a consolidated learning. In the following text the experience of implementing the constructivist approach to the teaching of legal medicine is described. Materials and methods. We used one of the workshops scheduled on the subject-prescription-to test the potential of this learning method in medicine and to determine its validity. Results and conclusions. The results show that the method in all its forms is particularly suited for the medical student and professional because it requires an efficient integration of information and the development of some assumptions which will put under a critical review, and will be applied in the daily practice of their profession.

Key words. Constructivist teaching method. Education. Medicine. Students.

\section{Introducción}

Clásicamente, el docente ha lamentado, en no pocas ocasiones, la limitación de tiempo que restringe los contenidos que puede o no puede impartir, y a menudo abandona el aula con la sensación de que ha tratado el tema de forma epidérmica.

A esta limitación secular ya constatada cuando impartimos una clase magistral, se debe añadir aquella limitación temporal con la que se encuentra el docente que pretende aplicar un método constructivista a sus enseñanzas.
Facultad de Medicina. U.D. Medicina Legal. Universidad de Valencia. Valencia, España.

Correspondencia Dr. Francesc Francés Bozal. Facultad de Medicina. U.D. Medicina Legal. Universidad de Valencia. Avda. Blasco Ibáñez, 15. E-46010 Valencia.

E-mail francesc.frances@uv.es 
Este tipo de enfoque [1,2], si bien ampliamente aceptado y aplicado en otras áreas de conocimiento, va implantándose lentamente en la formación médica. En este abordaje metodológico del aprendizaje, es el alumno el que va a construir el conocimiento sobre las bases cognitivas que ya posee. El profesor que sigue este método no se limita a suministrar un conocimiento ya elaborado, sino que ha de dirigir al alumno en su proceso autónomo y activo de aprendizaje. El alumno construye su propio conocimiento y la tarea del profesor es tutelar y orientar ese proceso de aprendizaje, frente al papel clásico de transmisor de conocimiento. Así, los diferentes métodos pedagógicos constructivistas coinciden en redefinir los roles de alumnos y profesor. Diversas experiencias han demostrado que el alumno que construye activamente su propio aprendizaje lo consolida y retiene mucho más eficazmente que si se ha limitado a recibir éste de forma pasiva, ya completamente elaborado por parte del profesor, sin necesidad de un esfuerzo por su parte.

Obviamente el alumno necesita una información previa y un tiempo de elaboración y procesamiento de esos datos para conseguir construir un aprendizaje duradero. Ése es el motivo de la gran limitación que a nuestro juicio tiene la implantación extensiva de este tipo de métodos en el ámbito de la docencia médica, tanto en pregrado como en posgrado.

\section{Materiales y métodos: nuestra experiencia}

La asignatura de Medicina Legal en la Facultad de Medicina de Valencia tiene un contenido de créditos teóricos y además se imparten tradicionalmente una serie de seminarios de tres horas que tratan de hacer hincapié en determinados aspectos de la asignatura que son de especial interés. Muchos de estos seminarios versan sobre aspectos medicolegales de inmediato impacto práctico sobre el médico de familia en ejercicio, como puede ser los documentos medicolegales, la carrera profesional, la prescripción médica, etc.

Así, hemos aprovechado la oportunidad que nos brindan los seminarios, no para suministrar de forma pasiva para el alumno conocimientos adicionales en forma de una clase magistral de tres horas, sino que hemos optado por intro-
Tabla. Contenidos.

Conceptuales

- Concepto de prescripción y de dispensación

- Marco legislativo sobre la prescripción

- Tipos de medicamentos a efectos de prescripción

Procedimentales

- Prescribir mediante el documento adecuado

- Obtener información acerca del medicamento (vademécum)

- Registrar adecuadamente los datos relativos a la prescripción:

- Datos del profesional

- Datos del enfermo

- Datos del medicamento

Actitudinales

- Valorar de forma crítica los factores limitadores de la libertad de prescripción del médico

- Sensibilización acerca de la necesidad de garantizar al paciente una prescripción libre

ducir, gracias a este tiempo extra, enfoques constructivistas en determinados contenidos que eran especialmente favorables a este método.

Nuestra experiencia ha consistido en reelaborar el seminario que se impartía bajo el título de 'La prescripción médica'. En él hemos hecho especial énfasis en la libertad de prescripción, conscientes de que, más allá de los aspectos formales de los diferentes tipos de receta médica y legislación sobre prescripción y dispensación, los médicos noveles se encuentran con limitaciones a la hora de prescribir y, caso de no saber identificarlas y enfrentarse adecuadamente a ellas, pueden representar un perjuicio para el profesional y para el paciente.

Así pues, se definieron los contenidos conceptuales, procedimentales y actitudinales (Tabla). En este artículo nos centraremos en la forma en la que se abordó el aprendizaje de los contenidos actitudinales.

El seminario de tres horas finalmente queda distribuido de la siguiente manera: primeramente se da a los alumnos una mínima introducción teórica sobre las bases éticas y científicas, formales y legales de la prescripción y se les familiariza con la cumplimentación del documen- 
to prescriptivo por excelencia, la receta médica. En esta primera parte, el alumno adquiere los contenidos conceptuales y procedimentales relativos a la prescripción (Tabla). Posteriormente indicamos a los alumnos que se organicen en grupos de 4-5 personas. Se les informa que van a tener que identificar de forma cooperativa aquellos factores que potencialmente pueden limitar la libertad de prescripción. En primer lugar deben definir los actores que intervienen en el acto médico de forma global para posteriormente identificar cuáles pueden limitar en algún caso su libertad a la hora de prescribir uno u otro medicamento. Para ayudarlos en esta tarea se les facilita una serie de artículos obtenidos de revistas de divulgación que, sin hacer referencia explícita al tema, incluyen aspectos relativos a la libertad de prescripción.

Después de un periodo breve de lectura, cada equipo debe abrir un debate para identificar las limitaciones con las que se encontrarán en el futuro a la hora de prescribir y en qué manera esta limitación se produce. Se les pide también que gradúen el potencial limitador de su libertad en cada caso, que consensúen un peso específico para cada factor limitante.

Posteriormente a un animado debate en el seno de cada grupo, el profesor les solicita que informen a los otros equipos de sus conclusiones escribiéndolas en la pizarra. Así se abre una nueva discusión, ya que si un grupo ha identificado un factor que otro no han percibido, el portavoz del primero explicará en voz alta el porqué de su inclusión.

Durante todo el proceso el profesor orienta la discusión que se produce primeramente en el seno de cada grupo y posteriormente en la puesta en común. Esta labor orientativa y de guía permite que los alumnos identifiquen facetas de limitación prescriptiva en las cuales no habían pensado. Es gratificante observar cómo los mismos alumnos se sorprenden de las formas insospechadas en las que determinados actores pueden influir en su prescripción. Así, se abre un nuevo debate sobre la polémica prescripción por el colectivo de enfermeros, los cambios de prescripción por parte del farmacéutico y las nuevas estrategias de ventas de los laboratorios farmacéuticos. Una vez el debate se ha abierto, el profesor enuncia el marco normativo de estos temas y los alumnos comentan abiertamente la situación.
Finalmente, mediante unas breves preguntas abiertas a toda la clase, comprobamos que el alumnado ha interiorizado los diferentes contenidos.

\section{Resultados y conclusiones}

En nuestra experiencia, hemos partido de una premisa constructivista a la cual hemos añadido un matiz de aprendizaje cooperativo, en la que la construcción del conocimiento por parte del alumno se enriquece con las opiniones del resto del grupo [3].

Fruto de la implantación de este método ha sido la constatación de que los alumnos todavía están muy poco familiarizados con estos métodos. El alumnado está escasamente acostumbrado a las técnicas de aprendizaje cooperativo, e inicialmente ve con extrañeza y recelo el hecho de tener que generar grupos y reorientar el mobiliario del aula. La comunicación en el seno de la clase cuesta de iniciar. Además, nuestro alumnado está poco familiarizado con la confrontación de opiniones y el debate, mostrándose en exceso individualista, fruto de una enseñanza clásica de predominio magistral. No obstante, cuando se hace evidente que ninguna opinión se censura y que toda visión aporta algo al grupo, la dinámica de colaboración crece de forma exponencial.

Por último, y relativo al tema de la prescripción médica, constatamos que la mayoría de alumnos -y podemos suponer que también los profesionales en formación- no se perciben a ellos mismos como un agente limitador de su propia libertad. Al identificar al propio facultativo como limitador, hacemos énfasis en la necesidad de la formación continua y la eventualidad de la acreditación periódica de conocimientos médicos.

A nuestro modo de ver, el método constructivista en cualquiera de sus modalidades es especialmente idóneo para el profesional médico porque obliga a una integración eficiente de la información recibida y a la elaboración de unos supuestos que serán sometidos a una revisión crítica. Educar al alumno universitario y al médico novel en esta forma de pensamiento y de construcción de conocimiento guarda una similitud inmediata con la práctica diaria de su profesión, en la que tendrá que interpretar críti- 
camente una información mediante la aplicación e integración de sus conocimientos previos para adquirir saberes que no posee o hacer frente a situaciones ante las cuales no se ha enfrentado todavía.

Es este el motivo por el que proponemos a los profesionales docentes y tutores de los médicos en formación en Medicina Familiar y Comunitaria la aplicación de estos abordajes de enseñanza, dada la necesidad de capacitar al médico para enfrentarse activamente a los problemas diarios en la consulta, mediante la provisión de un conocimiento no sólo conceptual y procedimental, sino también actitudinal, sólidamente adquirido, bien elaborado y estructurado, y con aplicación práctica inmediata.

\section{Bibliografía}

1. Piaget J. Psicología y epistemología. Buenos Aires: Emecé; 1972.

2. Coll CS. Aprendizaje escolar y construcción del conocimiento. Barcelona: Paidós; 1991.

3. Gillies, RM, Ashman, AF. Co-operative learning: the social and intellectual outcomes of learning in groups. London: Routledge Falmer; 2003. 